\title{
The Radiation-Initiated Polymerization of Styrene in Wax
}

\author{
A. C. Thomas, T. A. du Plessis, Anne Oosterhuis, \\ and Henriëtte OOSTERHUIS \\ Chemistry Division, Atomic Energy Board, Republic of South Africa.
}

(Received February 10, 1973)

\begin{abstract}
The presence of a paraffin wax has been found to considerably reduce the radiation dose required to completely polymerize styrene, but no detectable grafting of styrene to wax occurs. The rate of polymerization was found to be greatly dependent on the styrene/wax composition, and the activation energy was determined to be -7.4 $\mathrm{kcal} / \mathrm{mole}$. The addition of scavengers indicates a free-radical mechanism which is confirmed by preirradiation experiments, showing the wax to be the main source of initiating radicals. The negative activation energy is explained by an induced gel-effect caused by the semirigid wax matrix, which further enhances the polymerization rate, which cannot be accounted for entirely in terms of increased radical production in the wax.

KEY WORDS $\gamma$-Radiation / Styrene / Paraffin Wax / Radiation Polymerization /
\end{abstract}

In an earlier study ${ }^{1}$ attempting to graft vinyl monomers onto paraffin waxes by irradiation, it was found that, although grafting was not achieved, the waxes greatly enhances the polymerization of monomers which either were polymerized very slowly or not at all by radiation initiation under normal conditions, i.e., unless 'super-dry' or at low temperatures. These monomers include certain vinyl ethers as well as styrene which is of particular interest due to its low cost relative to other monomers in South Africa. Although styrene is readily polymerized by chemical initiation, the stability imparted to it by the phenyl group makes it particularly difficult for polymerization to occur by means of radiation initiation. A method for reducing the radiation dose required for this polymerization can be of practical importance. For this reason it was decided to investigate the styrene/ wax system in greater detail.

\section{EXPERIMENTAL}

The wax used for this study was Sasolwaks $\mathrm{M}^{*}$ (congealing temperature $57-60^{\circ} \mathrm{C}$ ). The styrene containing 70-ppm tertiary butyl catechol as an inhibitor was used as such for most experiments. In experiments using purified monomer,

\footnotetext{
* Kindly supplied by the Sasol Marketing Co. Ltd.
}

the commercial material was treated in the usual manner. $^{2}$

The reactants were degassed before being sealed in glass ampoules, after which the contents were heated to melt the wax to effect homogeneous dispersion of the monomer. The ampoules were irradiated at temperatures controlled to within $0.5^{\circ} \mathrm{C}$ in a ${ }^{60} \mathrm{Co}$ irradiation facility (Gammabeam 650, AECL). Dose-rate determinations were carried out by Fricke dosimetry. After irradiation, the contents of the ampoules were extracted in a Soxhlet apparatus for $24 \mathrm{hr}$, using petroleum ether $\left(60-80^{\circ} \mathrm{C}\right)$ to remove the wax and unpolymerized monomer from the polystyrene formed. The polymer was dried to constant weight to obtain the percentage conversion. Where deemed necessary, control experiments were carried out in which only the irradiation step was omitted, so that any polymerization due to the other procedures could be measured.

Molecular weight measurements were carried out by vapour-pressure osmometry, using toluene as the solvent at $50^{\circ} \mathrm{C}$, or by determination of intrinsic viscosities in the same solvent at $30^{\circ} \mathrm{C}$. In the latter method number-average molecular weights were obtained from the Mark-Houwink equation using constants determined by Danusso and Moraglio. ${ }^{3}$ 


\section{RESULTS}

\section{Polymerization Rate at Various Compositions}

Percentage conversions of styrene to polystyrene at various radiation doses were determined for styrene in wax contents, varying from 9 to $100 \%$ $(\mathrm{w} / \mathrm{w})$ at a fixed temperature $\left(25^{\circ} \mathrm{C}\right)$ and dose rate (1.5 Mrad/hr). The rate curves obtained exhibited no induction period and, initial polymerization rates were measured from the initial straight-line portions of these curves ( $<15 \%$ conversion). These values are plotted against their respective styrene contents in Figure 1, showing how markedly the styrene/wax ratio affects the polymerization rate.

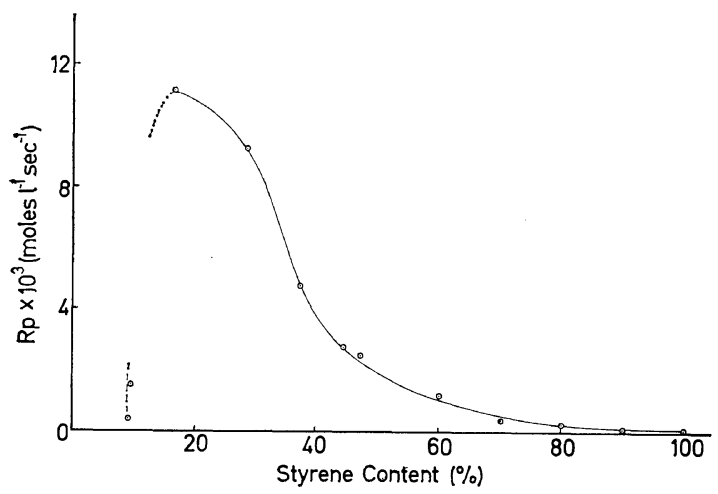

Figure 1. Initial polymerization rate as a function of percentage styrene content.

\section{Effect of Temperature on Polymerization Rate}

Conversion curves were obtained for two wax/ monomer compositions at various reaction temperatures and the rate obviously increased considerably with decreasing temperature. Initial rates were obtained from these curves to give the Arrhenius plots shown in Figure 2, from which it may be seen that a reasonably linear relationship exist in the temperature range from $-19^{\circ} \mathrm{C}$ to about $+30^{\circ} \mathrm{C}$, after which the rate falls off more rapidly. Such behaviour is believed to be due to softening of the wax as the temperature approaches its melting point, thereby altering the phase in which the polymerization occurs. The slopes of the straight-line portions of the curves yield values for the activation energy of the reaction of -7.8 and $-6.8 \mathrm{kcal} / \mathrm{mole}$ for the $29-\%$ and $38-\%$ mixtures respectively.

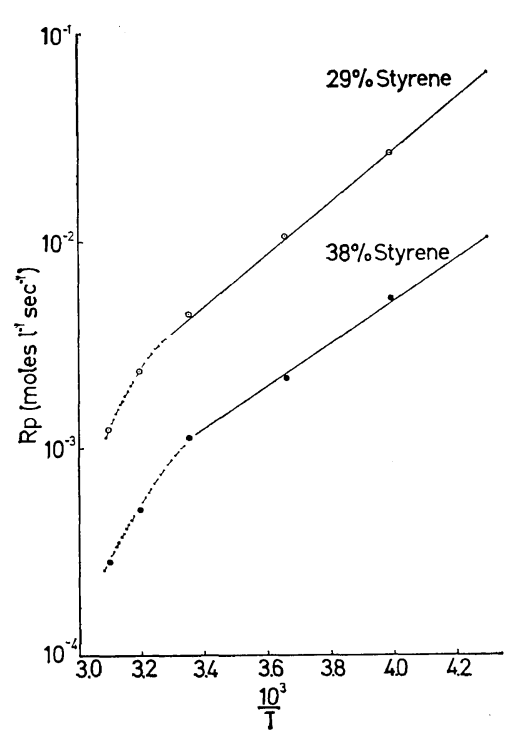

Figure 2. Temperature dependence of polymerization rate at different wax/monomer compositions.

\section{Effect of Radiation Intensity}

The dependence of the polymerization rate on the dose rate was briefly examined. Logarithmic plots gave a mean power dependence of 0.3 , giving little indication of the reaction mechanism involved. ${ }^{4}$

\section{Effect of Additives}

Table I shows the results of experiments in which various scavengers were added to the mixture. It may be noted that oxygen and benzoquinone almost completely inhibited the

Table I. Effect of additives on the polymerization ${ }^{a}$

\begin{tabular}{lccc}
\hline Additive & $\begin{array}{c}\text { Percentage } \\
\text { styrene } \\
\text { content }\end{array}$ & $\begin{array}{c}\text { Additive } \\
\text { concentration, } \\
\%(\mathrm{w} / \mathrm{w})\end{array}$ & $\begin{array}{c}\text { Percentage } \\
\text { conversion } \\
\text { to polymer }\end{array}$ \\
\hline None & 29 & - & 36.6 \\
Benzoquinone & 29 & 5.0 & 0.0 \\
Oxygen & 29 & Saturated & 0.9 \\
Water & 29 & 0.5 & 35.0 \\
Water & 29 & 1.0 & 34.7 \\
Methylene & 29 & 5.0 & 41.0 \\
chloride & 29 & 5.0 & 43.2 \\
Methanol & 29 & 71.0 & 5.2 \\
Heptane & 100 & - & 5.6 \\
None & 29 & Purified styrene & 45.9 \\
None & &
\end{tabular}

a Reaction conditions: temperature, $25^{\circ} \mathrm{C}$; total dose, 2.5 Mrad. 


\section{A. C. Thomas, T. A. Du Plessis, A. Oosterhuis, and H. Oosterhuis}

Table II. Comparison of the effect of different waxes on the polymerization ${ }^{2}$

\begin{tabular}{ccc}
\hline Percentage & \multicolumn{2}{c}{ Percentage conversion to polymer } \\
\cline { 2 - 3 } styrene content & M1 & L1 \\
\hline 29 & 100 & 42 \\
40 & 97 & 46 \\
45 & 96 & 41
\end{tabular}

a Reaction conditions: temperature, $25^{\circ} \mathrm{C}$ and total dose, 2.5 Mrad.

polymerization, whereas water, methanol and methylene chloride have little influence on the rate.

Heptane was used in one experiment in place of the wax, in order to ascertain the effect of a liquid hydrocarbon, but no increase in rate was achieved. A number of experiments were carried out to determine the extent of inhibition by the tertiary butyl catechol present in the commercial monomer. Table I gives the result of one such experiment, showing that the inhibited styrene exhibits a polymerization rate which is approximately $80 \%$ of that of the purified styrene.

Finally, the effect of using a softer wax was investigated. Sasolwaks L1 (congealing temperature $40^{\circ} \mathrm{C}$ ) was chosen. The results given in Table II, show that the softer wax is less than half as efficient as the M1 wax in 'promoting' the polymerization.

\section{Molecular Weight}

Table III lists values for the number-average molecular weight of polymer produced at various reaction temperatures. The values decrease with increasing temperature, with the exception of the $50^{\circ} \mathrm{C}$ value. Table IV shows the much lower molecular weights exhibited by polystyrene pro-

Table III. Molecular weight of polymer produced at different temperatures ${ }^{\mathrm{a}}$

\begin{tabular}{cc}
\hline Temperature, ${ }^{\circ} \mathrm{C}$ & $\bar{M}_{n}$ \\
\hline-19 & $1.7 \times 10^{6}$ \\
0 & $1.3 \times 10^{6}$ \\
25 & $6.0 \times 10^{5}$ \\
40 & $3.0 \times 10^{5}$ \\
50 & $3.5 \times 10^{5}$ \\
\hline
\end{tabular}

a Reaction conditions: styrene content 29\%; percentage conversion, $25 \%$.
Table IV. Comparison of polymerization rate and molecular weight of polymers produced in the presence and absence of wax

\begin{tabular}{cccc}
\hline $\begin{array}{c}\text { Percentage } \\
\text { styrene } \\
\text { content }\end{array}$ & $\begin{array}{c}\text { Dose, } \\
\text { Mrad }\end{array}$ & $\begin{array}{c}\text { Percentage } \\
\text { conversion }\end{array}$ & $\bar{M}_{n}$ \\
\hline 44 & 0.25 & 15 & $4.3 \times 10^{5}$ \\
29 & 0.25 & 29 & $5.9 \times 10^{5}$ \\
29 & 0.125 & 15 & $7.4 \times 10^{5}$ \\
100 & 7.5 & 8 & $4.2 \times 10^{3}$ \\
100 & 10.0 & 10 & $3.9 \times 10^{3}$ \\
100 & 20.0 & 21 & $3.6 \times 10^{3}$ \\
100 & 30.0 & 26 & $4.8 \times 10^{3}$ \\
\hline
\end{tabular}

duced by radiation initiation in the absence of wax and, also the greatly increased rate of polymerization achieved in the presence of the wax.

\section{Preirradiation Experiments}

These experiments were carried out in order to determine whether free radicals produced by radiolysis of the wax contributed significantly to the polymerization of styrene. Finely powdered wax was degassed for several hours before sealing it in ampoules and irradiating it to $5 \mathrm{Mrad}$ at $-196^{\circ} \mathrm{C}$. Deaerated styrene was then distilled onto the wax, and the contents of the ampoule allowed to warm slowly to room temperature. A $45-\%$ conversion of styrene to polystyrene was obtained.

\section{DISCUSSION}

The results show that the presence of paraffin wax has a pronounced effect on the radiationinduced polymerization of styrene. Three proposals can be put forward to explain this radiationenhanced polymerization rate, as compared to bulk styrene:

(i) the occurrence of an ionic mechanism

(ii) an energy transfer process from the wax to styrene

(iii) an induced gel-effect due to the restricting effect of the wax on molecular motion.

Ionic polymerization mechanisms are known to give fast reaction rates and high molecular weight products. Styrene undergoes polymerization by such a mechanism using radiation initiation, but generally only when the monomer is thoroughly dried, or at low temperatures. ${ }^{4,5}$ It has been suggested, however, that the inhibiting 
effect of water occurs only with solvated or clustered water molecules, ${ }^{4}$ or at least with dimerized water molecules. ${ }^{6}$ Du Plessis has proposed that methylene chloride reduces the inhibiting effect of water on the polymerization of isobutyl vinyl ether ${ }^{7}$ by destroying such an association of water molecules. It is conceivable that the wax in this case performs a similar duty, thus enabling an ionic mechanism to achieve the polymerization rates observed.

The most reliable indication of the mechanism involved is provided by the experiments involving additives (Table I). Benzoquinone and oxygen are well-known radical scavengers and their effect is clearly to prohibit the polymerization, whereas these compounds generally have little or no effect on ionic processes. As mentioned above, water, even in trace quantities, can have a profound effect on ionic mechanisms. No attempt was made in these experiments to dry the monomer, yet fast polymerization of styrene occurred, and even the addition of a relatively large amount of water had a negligible effect on the rate. Methylene chloride has frequently been used as a medium for carrying out ionic polymerizations, ${ }^{7,8}$ because of its high dielectric constant and nonpolar character. Methanol, on the other hand, interferes with and so retards such polymerizations. ${ }^{8}$ Neither of these additives, however, has a significant effect on the rate in this system. All the indications, therefore, point to a free-radical mechanism being responsible for the polymerization in question.

The results of the preirradiation experiments strongly corroborate the evidence for a free-radical mechanism, and also show that the free radicals formed by absorption of gamma rays in the wax are largely responsible for initiating the polymerization. The main reactions may thus be written

$$
\begin{aligned}
(\text { Wax }) \mathbf{W} & \rightarrow \mathbf{W} . \\
\mathbf{W} \cdot+(\text { Styrene }) \mathbf{S} & \rightarrow \mathrm{W}+\mathbf{S} . \\
\mathbf{S} \cdot+\mathbf{S} & \rightarrow(\mathbf{S})_{n}-\mathbf{S} .
\end{aligned}
$$

The role of the wax may thus be compared to that of various backbone polymers in the grafting reactions of styrene, ${ }^{9-11}$ and such behaviour accounts for the readiness of styrene to undergo radiation-induced graft polymerization as compared to its bulk-phase homopolymerization. In
Table V. Comparison of $G$-values and degree

\begin{tabular}{|c|c|c|c|}
\hline & \multirow{2}{*}{$\begin{array}{c}\text { Styrene } \\
\text { in bulk } \\
25^{\circ} \mathrm{C}\end{array}$} & \multicolumn{2}{|c|}{$\begin{array}{l}\text { Styrene in } \\
\text { wax, } 29 \%\end{array}$} \\
\hline & & $25^{\circ} \mathrm{C}$ & $-19^{\circ} \mathrm{C}$ \\
\hline$G(-$ monomer $)$ & 88 & $1.1 \times 10^{4}$ & $48.2 \times 10^{4}$ \\
\hline$\overline{D P}_{n}$ & 37.5 & $7.1 \times 10^{3}$ & $2.3 \times 10^{4}$ \\
\hline$G($ polymer $)=\frac{G(- \text { monomer })}{\overline{D P}_{n}}$ & 2.4 & 1.6 & 3.6 \\
\hline$G(\mathrm{R} \cdot)$ & $0.6^{13}$ & $6.0^{14, \mathrm{a}}$ & 6.0 \\
\hline$\frac{G(- \text { monomer })}{G(\mathrm{R} \cdot)}$ & $1.3 \times 10^{2}$ & $1.8 \times 10^{3}$ & $1.4 \times 10^{4}$ \\
\hline
\end{tabular}
of polymerization for styrene in bulk and in the presence of wax

a By comparison with higher alkanes.

this case, however, no grafting of styrene onto the wax could be detected. ${ }^{1}$ This indicates that the chain-transfer processes play an important role, resulting in the formation of homopolymer, as clearly shown by the preirradiation experiments.

Attempts to obtain telomers by radiolysis of styrene in various alcohols have failed to produce any trace of telomers, ${ }^{12}$ but instead polystyrene forms readily. This is indicative of the readiness of styrene to polymerize once initiation occurs via free-radical formation by the solvent.

Table V, however, shows that the rate of monomer conversion cannot be explained solely in terms of the rate of radical production in the different substrates, since the rate of conversion of monomer per radical formed is more than ten times greater in wax at $25^{\circ} \mathrm{C}$ than in bulk styrene, and a power of two greater in wax at $-19^{\circ} \mathrm{C}$, even assuming 100 per cent efficiency for wax radicals in initiating polymerization. This phenomenon is ascribed to the third proposal above, i.e., an induced gel-effect caused by the semirigidity of the wax matrix, which restricts molecular motion and hence mutual recombination of the growing polymer chains. The polymerization rate is therefore much enhanced, as is the degree of polymerization, as shown in Table V.

Presence of the proposed gel-effect is supported by the deviation from linearity which occurs in the Arrhenius plot (Figure 2) as described in section B of the results. It should be noted that the measured activation energy should be considered only as an apparent activation energy, since it is determined by several factors such as 


\section{A. C. Thomas, T. A. du Plessis, A. Oosterhuis, and H. Oosterhuis}

the degree of rigidity of the substrate and the mobility of the monomer and growing chain in this substrate. The mixture became noticeably less rigid as the proportion of styrene was increased, resulting in the sharply decreasing polymerization rate shown in Figure 1 above 20-\% styrene content. This effect is comparable to the reduction in the rate caused by softening of the substrate on increasing the temperature, and underlines the important role played by the rigidity of the matrix in enhancing the polymerization rate. This is further borne out by the lower efficiency of softer waxes in accelerating the polymerization, as shown in Table II, and by the absence of any enhancement of rate in the case of a liquid hydrocarbon (heptane).

In conclusion, it is proposed that the accelerated rate of polymerization of styrene in the presence of the wax occurs by a free-radical mechanism in which initiation is mainly by free radicals formed by radiolysis of the wax. The wax further increases the polymerization rate by limiting molecular mobility, and hence the deactivation of growing polymer chains by mutual recombination.

\section{REFERENCES}

1. A. C. Thomas, T. A. du Plessis, Anne Ooster- huis, and Henriëtte Oosterhuis, J. Appl. Polym. Sci., 17, 2519 (1973).

2. R. Y.M. Huang, J.F. Westlake, and S.C. Sharma, J. Polym. Sci., Part A-1, 7, 1729 (1969).

3. F. Danusso and G. Moraglio, ibid., 24, 161 (1957).

4. F. Williams, "Fundamental Processes in Radiation Chemistry," P. Ausloos, Ed., Interscience, New York, N.Y., 1968, p 515.

5. R. Y. M. Huang and J. F. Westlake, J. Polym. Sci., Part A-1, 8, 49 (1970).

6. M. Szwarc, Advances in Chemistry Series, 91, 236 (1969).

7. T. A. du Plessis, PEL 218, Atomic Energy Board, Pretoria (1971).

8. A. Chapiro, "Radiation Chemistry of Polymeric Systems," Interscience, New York, N.Y., 1962, p 308.

9. V. Stannett, K. Araki, J. A. Gervasi, and S. W. Mc Leskey, J. Polym. Sci., Part A, 3, 3763 (1965).

10. S. Machi, I. Kamel, and J. Silverman, ibid., Part A-1, 8, 3329 (1970).

11. T. Takamatsu and K. Shinohara, ibid., Part A-1, 4, 197 (1966).

12. L. van Wyk, private communication.

13. Ref $8, \mathrm{p} 82$.

14. Ref $8, \mathrm{p} 173$. 